\title{
Sub-mucosal micro-screws as ideal temporary anchorage devices
}

\author{
Amir-André Doustkam ${ }^{1,2, *}$, Sylvia Riemenschneider-Chillès ${ }^{3}$ \\ ${ }^{1}$ Oral Surgery and Oral Medicine Dentistry Department, Centre Hospitalier Régional de Colmar, Colmar, France \\ 2 Université de Strasbourg, Strasbourg, France \\ ${ }^{3}$ Oral Medicine and Surgery Service of CHU de Strasbourg, Strasbourg, France
}

(Received: 14 November 2016, accepted: 28 March 2017)

Keywords: micro-screw / orthodontic movement / bone anchorage

\begin{abstract}
Introduction: Tooth movements require a stable base. The most stable endo-buccal base is bone thus temporary bone-anchoring techniques have been well developed to date. Literature review of different boneanchoring techniques reveals limitations in their regular use, particularly when it comes to mini-screw stability. The purpose of this paper is to present a reliable temporary bone-anchoring technique pushing the limits of orthodontic treatments. Technique: The device is made of one to two micro-screws connected to a steel or titanium molybdenum alloy $\left(\mathrm{TMA}^{\oplus}\right.$ ) wire going through one or two loops. The device is then inserted into the bony base after a mucoperiosteal incision. The connecting arm, which emerges through the mucosa, is then connected to the teeth to be pulled in a direct or indirect manner. Comments: This device provides advantages in terms of reliability, versatility of orthodontic movements, simplicity in terms of protocol installation and removal, and poses no anatomical risk. Conclusion: This is an orthodontic surgical technique that provides a real benefit to the patient.
\end{abstract}

\section{Introduction}

Temporary bone-anchoring devices have revolutionized biomechanics, and consequently, orthodontic treatment [1]. Mini-screws are currently the most used temporary boneanchoring devices, with a clinical use of more than 20 years.

Despite their increasing use, the failure rate rises to $13.5 \%$ according to Papageorgiou's 2012 literature review [2]. Whether it is the mini-screws reliability rate, the relative complexity of the surgical protocol for mini-plates, the overall cost or more, temporary bone anchors are not ideal because they each have defects limiting their possibilities of use $[3,4]$.

\section{Technical}

\section{The micro-screws}

The micro-screws are osteosynthesis screws measuring $5 \mathrm{~mm}$ in length by $2 \mathrm{~mm}$ in diameter, intended to be placed under the gingival mucosa [5]. They are thread-forming, selftapping, and self-gripping, allowing one-handed insertion with an appropriate screwdriver. They are made of TiAl6V4 titanium alloy, ensuring their biocompatibility [6]. They do not have screw heads or transmucosal parts, unlike mini-screws, because

\footnotetext{
*Correspondence: amirandredoustkam@gmail.com
}

they are screwed directly into the depth of the vestibule with a connector which will emerge remotely from the screw insertion site.

\section{The connectors}

The connectors are composed of a steel or titanium molybdenum alloy (TMA) wire with a circular cross-section of $0.45 \mathrm{~mm}$ (0.018 inches) in diameter. They form one or two loops through which the screw run. As a result, the loop(s) will block against the cortical bone. At the other extremity of the loop(s), one or two arms will emerge in the oral cavity through the free mucosa.

The anchoring device consists of the micro-screw and connector set.

Connector classification depends on:

The material used (steel or TMA): "S" for steel (Steel), "T" for TMA. TMA can be employed as a resort for active direct anchoring, whereas steel has a rather passive direct or indirect anchoring role.

The number of loops: " 0 "-shaped for a single loop, " 8 "shaped for two loops. In the case of two micro-screws placed across " 8 " loops, true control in the three dimensions of the space of orthodontic movement will be possible. In fact, the distal micro-screw allows a fixed anchoring point to stabilize the connector, and the more mesial micro-screw ensures the direction of anchoring. 


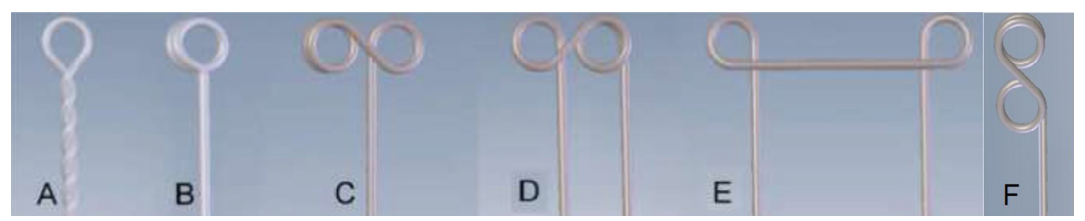

Fig. 1. Connectors CLO (A); CTO/CSO (B); CT8-1/CS8-1 (C); CT8-2/CS8-2 (D); CT8-2/CS8-2 tunnel (E); CT8-1/CS8-1 longitudinal (F) (adapted from Chillès et al. [5]).

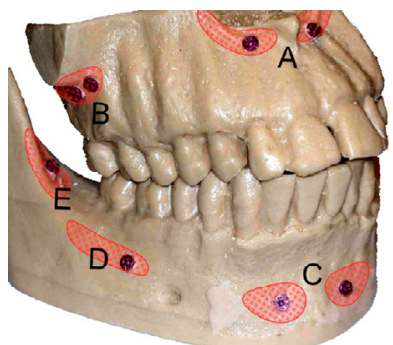

Fig. 2. Implantation sites (A) bottom edge of the piriform orifice; (B) zygomatic process of the maxilla; (C) mandibular symphysis; (D) external oblique line and; $(E)$ anterior edge of the ramus (adapted from Chillès et al. [5]).

The number of emerging arms: 1 or 2. Two arms will allow two independent actions or the connection with several teeth (Fig. 1).

\section{Implantation sites}

They are all extra-alveolar and at the depth of the vestibule because the cortical bone is thick at this level. They are also distant from anatomical elements at risk (dental roots, nasal cavities, sinus, and inferior alveolar nerve). Special attention must be paid to the mental foramen because insertion is avoided in this area and in the lower alveolar channel according to the radiological study of Safaee et al. [7], which showed that the micro-screws are shorter than the distance between the buccal cortical and the lower alveolar canal. Five sites are thus available (Fig. 2).

\section{The orthodontic surgical protocol}

After intraoral decontamination by an antiseptic solution, local anesthesia is administered slightly away from the application site.

Then, a mucoperiosteal incision of $10 / 12 \mathrm{~mm}$ is placed using a scalpel blade \#15 at the depth of the vestibule in the free mucosa (Fig. 3A).

The periosteum is then peeled off using a periosteal elevator at a depth of approximately $5 \mathrm{~mm}$ to expose the underlying bone (Fig. 3B).

The screw at the end of the screwdriver is threaded through the loop on the chosen connector, and the set is held with one hand only (Fig. 3C). The installation of the screw is made freehand with the dedicated screwdriver.
Table I. Qualitative comparison between mini-screw and micro-screw devices.

\begin{tabular}{lll}
\hline Ideal anchoring criteria & \multicolumn{2}{c}{ Mini-screws Micro-screw } \\
& & system \\
\hline Simplicity of the protocol & $\checkmark$ & $\checkmark$ \\
Anatomic risks & exist & don't exist \\
Versatility of orthodontic movements & $\checkmark$ & $\checkmark$ \\
Versatility of connectors & $\checkmark$ & $\checkmark$ \\
Cost & Moderate & Moderate \\
Immediate loading & $\checkmark$ & $\checkmark$ \\
Space requirement & Low & Low \\
3D control/independent device & $\times$ & $\checkmark$ \\
\hline
\end{tabular}

Its rotation is effected through a wheel located between the forefinger and the thumb. The screw-introduction axis is perpendicular to the bone surface and it does not require predrilling (Fig. 3D).

The connector is released during the introduction of the screw into the cortical bone. In the case of an " 8 " loop, the mesial screw is placed first.

Once the connector starts to be driven by the rotation of the screw, the latter is slightly loosened. The wire is repositioned and then maintained during final tightening or the placement of a screw pair (in the case of " 8 " loops).

The initial stability of the screw is controlled manually.

At this point in the intervention, a bayonet is prepared with an orthodontic "push spring" at the level of the incision line, allowing the emergence of the connector arm in the oral cavity (Fig. 3E and F). The endpoint of the connector is performed, in this case in a hook for connection to the orthodontic arch by two elastic chains (Fig. $3 \mathrm{G}$ and $\mathrm{H}$ ).

The ends of the connectors are versatile because they are molded directly in the mouth depending on the choice and according to the clinic that will manage their molding, for a full congruence between the teeth and the connectors.

The procedure requires no stitches.

An immediate postoperative orthopantomogram allows the visualization of the position and the axis of the screws.

A simple prescription, including a level- 1 analgesic as well as chlorhexidine mouthwash, is made. No antibiotic prescription is necessary at first, in accordance with current recommendations concerning implant devices (MSNA 2011) [8]. 

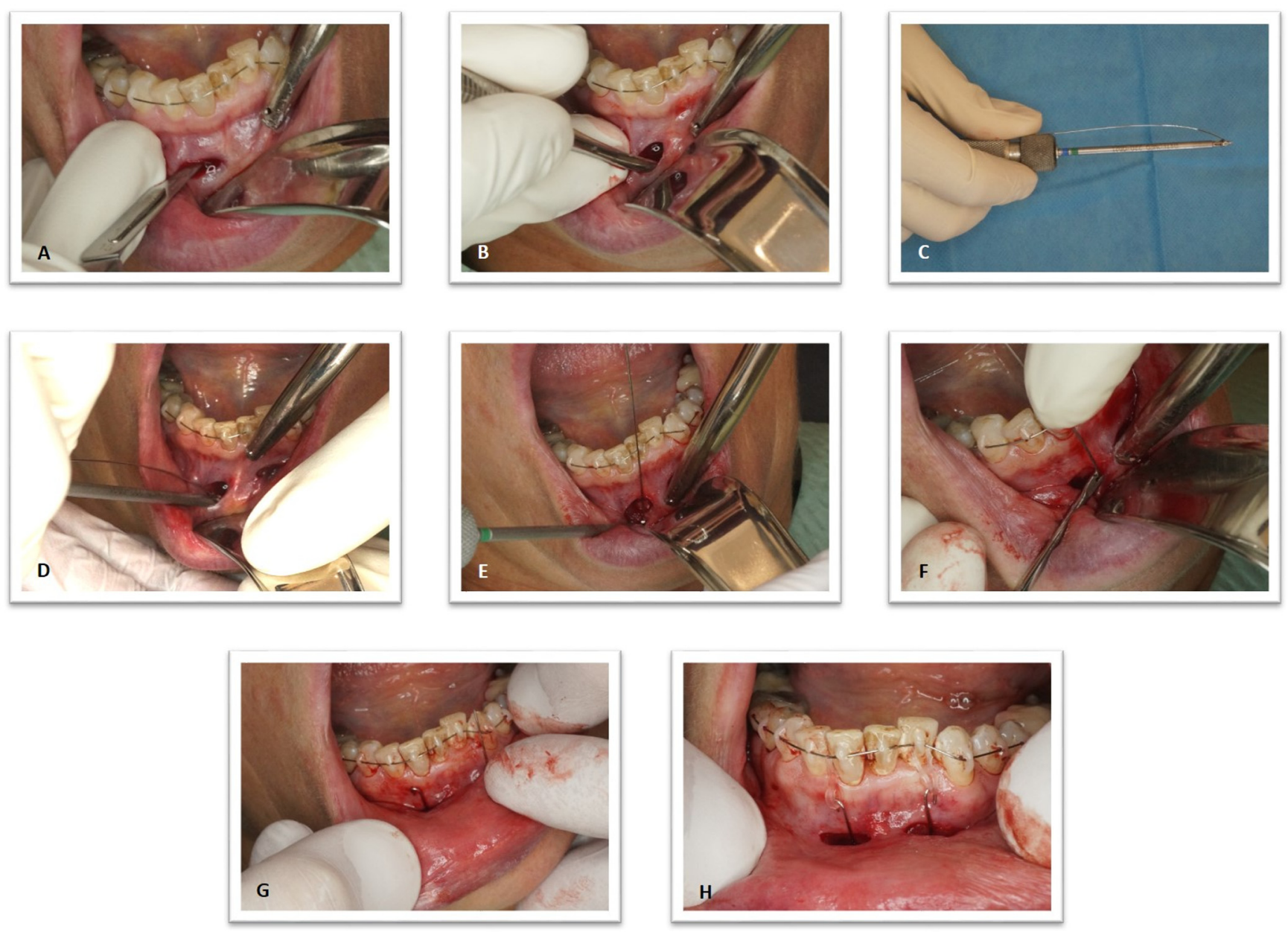

Fig. 3. (A) Incision, note the tension on the labial mucosa and the scalpel axis; (B) periosteal elevation with the stripper; (C) retention of connectivity and presentation of the screw at the end of the screwdriver before insertion; (D) insertion of the device; (E) stabilization of the connector arm against the bone; $(F)$ preparation of a bayonet to bring out the connection arm in the free mucosa; $(G)$ realization of a hook at the termination of the connection and; $(\mathrm{H})$ connecting the device to the orthodontic arch wire by a rubber band ligation (courtesy: Dr. Chillès).

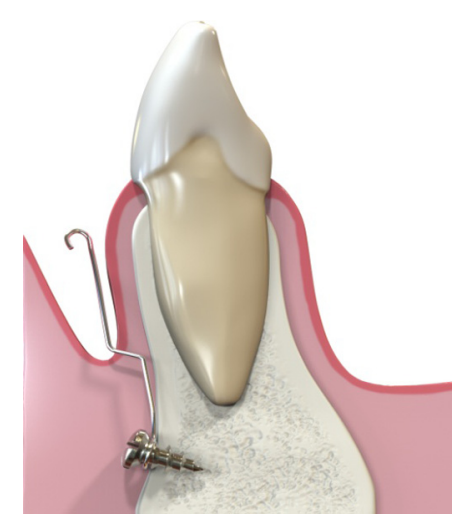

Fig. 4. Schematic representation of the insertion of the micro-screw device. Note the folding away from the screw tip and the emergence of the connector arm in the free mucosa.

Brushing of the surgical site after the emergence of connector, using a $7 / 100$ surgical toothbrush soaked in chlorhexidine is performed twice a day, starting $48 \mathrm{~h}$ after the surgery and for the whole treatment duration.
Removal protocol, once the orthodontic action is completed, requires a new incision under local anesthesia. If the microscrews were not osseointegrated, removal is easy. It is sometimes necessary to cut submucosal scar fibrosis to access the screw head.

\section{Comments}

One of the key points of the stability of the temporary bone anchoring is to minimize the inflammatory risk around the screws [9-12].

The initial mucoperiosteal incision and periosteal elevation allows to position the micro-screws directly against the bone. Because of this, the gums can heal over the screw head and the connector loop. The free mucosa will then act as a protective barrier against physical and chemical attacks of the oral cavity, as observed by Park et al. for the mini-screws covered in undesirable ways in the free mucosa [13].

The bayonet prepared allows the emergence of the connector through this protective free mucosa. The risk of screw loss because of bacterial infection, an argument 
advanced by Cheng et al., becomes negligible for microscrews, because the distance between their submucosal head and the emergence of the wire strongly decreases the risk of bacterial infiltration at the bone level (Fig. 4) [14].

For mini-screws, cortical thickness of $1.4 \mathrm{~mm}$ would be required to ensure primary stability according to Hibi et al. [15]. In our study the chosen sites fulfill this condition.

The micro-screw system ranks among the most reliable temporary bone-anchoring systems according to the reported results. One study reported a loss of 15 devices (10 micro-screw losses and five archwire fractures) or a $96.8 \%$ overall success rate (95\% CI [94.5-98.1]) on a series of consecutive cases of 264 patients for whom 434 devices were implanted with 644 micro-screws, after a minimum of 12 months in use and a maximum of 16 years follow-up [16].

As the micro-screws are short in length and located on a bony base, there is no risk of injury to dental roots or anatomical structures at risk, such as the lower alveolar nerve. In addition, there is no risk of interference with dental movement.

Biomechanically, as the wire is plated against the bone, there is almost no lever arm that could displace the screw. According to the study of Holmgren et al., when a lateral load is applied on a dental implant, the force is concentrated on the top of the screw and distributed to the bone [17]. Holmgren et al. also reported a positive correlation between the cortical bone thickness and the breakout force [18]. The insertion sites of the micro-screws feature relatively thick cortical as it forms the bony base which explains the resistance of the microscrews.

As the incision is small, there is no need to suture to obtain an hemostatic benefit, which may predispose to local inflammation by plaque retention [19].

The use of double loops (CT8/CS8 system) offers the possibility of control in three-dimensional space. The distal micro-screw allows the stabilization of the connector, by avoiding the rotation of the loop working around the mesial micro-screw.

The use of the micro-screw system has the advantage to treat only the necessary teeth, and so not to necessarily mobilize the other teeth. In addition, thanks to the widereaching arm and three-dimensional information it can initially store, the micro-screw system is independent.

The table below summarizes the advantages of the microscrew device compared with anchoring mini-screws (Tab. I).

\section{Conclusion}

The micro-screw device seems to meet the conditions of absolute ideal bone anchoring:

- stability over the treatment duration;

- simplicity of the implantation and removal protocols;

- decreased size for ubiquitous insertion;

- absence of anatomical risk;

- free orthodontic movement;

- moderate cost:

- possibility of immediate loading;
- space saving ability;

- versatility of connector ends.

Here is an example of orthodontic surgical coordination offering real clinical benefit for the patient.

Conflicts of interests: The authors declare that they have no conflicts of interest in relation to this article.

\section{References}

1. Peuch-Lestrade G, Le Marie M, Guillaumot G, Decker A. Les minivis en orthodontie. Int Orthod 2009;7:157-169.

2. Papageorgiou SN, Zogakis IP, Papadopoulos MA. Failure rates and associated risk factors of orthodontic mini screw implants: a meta-analysis. Am J Orthod Dentofac Orthop 2012;142:577-595.

3. Chillès $D$, Chillès JG. Introduction à l'utilisation de vis de chirurgie maxillo-faciale comme ancrage orthodontique. Rev Orthop Dento-Fac 2006;40:63-90.

4. Chillès D, Chillès JG. Un dispositif à ancrage squelettique pour la traction et la mise en place des canines incluses. Rev Orthop Dento-Fac 2009;43:67-86.

5. Chillès JG, Riemenschneider-Chillès $S$. Utilisation des minivis corticales courtes en orthodontie. Orthod Fr 2011;82:253-268.

6. Nosouhian S, Rismanchian M, Davoudi A. A mini-review on the effect of miniimplants on contemporaryorthodontic science. J Int Oral Health 2015;7 (Suppl 1):83-87.

7. Safaee A, Mirbeigi S, Ezoddini F, Khojastepour L, Navab-Azam A. Buccolingual course of the inferior alveolar canal in different mental foramen locations: a cone beam computed tomography study of an Iranian population. Int $\mathrm{J}$ Appl Basic Med Res 2016;6:262-266.

8. Agence Française de Sécurité Sanitaire des Produits de Santé. Prescription des Antibiotiques en Pratique Bucco-Dentaire: ANSM, 2011. Available from: http//ansm.sante.fr/Mediathe que/Publications/Recommandations-Médicaments.

9. Moon C-H, Lee D-G, Lee H-S, Im J-S, Baek S-H. Factors associated with the success rate of orthodontic mini screws placed in the upper and lower posterior buccal region. Angle Orthod 2008;78:101-106.

10. Antoszewska J, Raftowicz-Wójcik K, Kawala B, MatthewsBrzozowska T. Biological factors involved in implant-anchored orthodontics and in prosthetic-implant therapy: a literature review. Arch Immunol Ther Exp (Warsz) 2010;58:379-383.

11. Wu T-Y, Kuang S-H, Wu C-H. Factors associated with the stability of mini-implants for orthodontic anchorage: a study of 414 samples in Taiwan. J Oral Maxillofac Surg 2009;67:1595-1599.

12. Kuroda S, Sugawara Y, Deguchi T, Kyung H-M, Takano-Yamamoto T. Clinical use of mini screw implants as orthodontic anchorage: success rates and postoperative discomfort. Am J Orthod Dentofacial Orthop 2007;131:9-15.

13. Park HS, Jeong SH, Kwon OW. Factors affecting the clinical success of screw implants used as orthodontic anchorage. Am J Orthod Dentofacial Orthop 2006;130:18-25.

14. Cheng S, Tseng I, Lee J, Kok S. A prospective study of the risk factors associated with failure of mini-implants used for orthodontic anchorage. Int $\mathrm{J}$ Oral Maxillofac Impl 2004;19:100-106.

15. Hibi H, Sakai K, Oda T, Hattori H, Ueda M, Sakai M. Stability of a locking plate and self-drilling screws as orthodontic skeletal anchorage in the maxilla: a retrospective study. J Oral MaxillofacSurg 2010;68:1783-1787. 
16. Doustkam AA. Les micro-vis sous muqueuses comme ancrage osseux temporaire en pratique orthodontique: Étude longitudinale sur 16 ans. Thèse de Doctorat en médecine, Faculté de médecine de Strasbourg, $n^{\circ}$ 104, 2016.

17. Holmgren E, Seckinger R, Kilgren L, Mante F. Evaluating parameters of osseointegrated dental implants using finite element analysis a two dimensional comparative study examining the effects of implant diameter, implant shape, and load direction. J Oral Implantol 1998;24:80-88.
18. Migliorati M, Drago S, Schiavetti I, Olivero F, Barberis F, Lagazzo A, Cappuro M, Silvestrini-Biavati A, Benedicenti S. Orthodontic miniscrews: an experimental campaign on primary stability and bone properties. Eur J Orthod 2015;37:531-538.

19. Leknes KN, Selvig KA, Boe OE, Wikesjo UME. Tissue reactions to sutures in the presence and absence of anti-infective therapy. $J$ Clin Periodontol 2005;32:130-138. 